\title{
Handbook of Clinical Anaesthesia, Fourth Edition
}

\author{
Brian J. Pollard, Gareth Kitchen (Eds). CRC Press; 2017, 784 pages. Kindle edition: \\ \$125.70 CAD, hardcover: \$306.2 CAD, paperback: \$126.70 CAD. ISBN-10: 1498762891, \\ ISBN-13: 978-1498762892
}

\author{
Is'haq Al Aamri, MD, BHSc
}

Received: 2 August 2018/Accepted: 2 August 2018/Published online: 22 August 2018

(C) Canadian Anesthesiologists' Society 2018

The Handbook of Clinical Anesthesia, Fourth Edition offers senior trainees and practicing anesthesiologists a concise review of current anesthesia practice. It was originally published in 1996, and the last edition appeared in 2011. It is a multi-authored textbook with contributions exclusively from the United Kingdom (UK).

The book is organized into three sections. The first section, Patient Conditions, consists of nine chapters dedicated to various organ systems. These chapters cover the respiratory, cardiovascular, central nervous, and endocrine systems; the genitourinary and gastrointestinal tracts; blood, bone, and joints; and connective tissue disorders. Each chapter reviews the relevant disease processes, including epidemiology, pathophysiology, disease burden, and ongoing clinical management, concluding with patient optimization for anesthesia and surgery.

The second section, Surgical Procedures, consists of 14 chapters covering abdominal, gynecologic, obstetric, thoracic, cardiac, vascular, ophthalmic, ear nose and throat, head and neck, plastic surgery, as well as neurosurgery, orthopedics, urology, transplantation, and pediatrics. Each chapter reviews specific anesthetic considerations, including preoperative assessment and optimization; perioperative management including intraoperative anesthesia care; and postoperative issues including pain control. In addition, each chapter reviews the advantages and disadvantages associated with the various types of anesthesia care offered (i.e., general, neuraxial, regional, local, and/or intravenous sedation).

The third section, Anesthetic Factors, is divided into six chapters that cover the topics of preoperative assessment,

I. Al Aamri, MD, BHSc ( $\square)$

McGill University, Montreal, QC, Canada

e-mail: ishaq.alaamri@mail.mcgill.ca the airway, equipment and monitoring, general techniques, regional techniques, and management problems. The latter chapter reviews an extensive range of topics including various intraoperative emergency crises that require attention (e.g., malignant hyperthermia/ neuroleptic malignant syndrome, bronchospasm, local anesthetic toxicity, total spinal anesthesia, TURP syndrome, allergic reaction, embolism, and Advanced Cardiovascular Life Support), fluid and electrolyte balance (including blood transfusion), patient positioning, awareness, delayed awakening, robotic surgery, major trauma, pacemakers, electroconvulsive therapy, pseudocholinestrase deficiency, elevated intracranial pressure/cerebral blood flow control, robotic surgery, and transfer of the critically ill patient.

Editors Pollard and Kitchen offer a concise summary of anesthesia care in this Handbook. The chapters conclude with a small number of references for additional information, and overlapping topics are identified as "cross references" to help link the information. Given its scope, the book is appropriately comprehensive. Its exclusive UK authorship of this text, however, makes it somewhat awkward for use by North American anesthesia providers. For instance, acronyms (e.g., HDU = high dependency unit), drug names (e.g., paracetamol vs acetaminophen), and regulatory bodies (e.g., AAGBI = Association of Anaesthetists of Great Britain and Ireland) may not be familiar to North American readers. Moreover, drugs that are commonly used in the UK may not be currently in use in North America (e.g., "thiopental is the drug most commonly used for induction in the UK"). A notable shortcoming is the lack of color in the text's photographs, tables, algorithms, and illustrations so that key messages may not be clearly emphasized. The Techniques (regional) chapter briefly summarizes only basic information, and important details, such as 
anatomical illustrations, optimal drug dosing, and delivery systems, are not provided. Moreover, although allusions to ultrasound are made, no ultrasound images are provided. Also, the book advertises as a "Handbook", although its relatively large size $(25 \times 20 \mathrm{~cm}, 1.6 \mathrm{~kg})$ suggests otherwise.

In conclusion, this Handbook of Clinical Anaesthesia, Fourth Edition is a well-organized, easy-to-read product suitable for trainees in anesthesia and practicing anesthesiologists looking for a clinical reference that efficiently covers key concepts. I would recommend this high-yield book to practitioners seeking to build and maintain a foundation of knowledge in clinical anesthesia care. Nevertheless, a future edition would do well to address the difficulties that anesthesia providers trained outside the UK may encounter while using this textbook. Moreover, the basic nature of the accompanying graphics (black and white, only) requires updating along with consideration to incorporate web-based learning and multimedia content.

Conflicts of interest None declared.

Editorial responsibility This submission was handled by Dr. Hilary P. Grocott, Editor-in-Chief, Canadian Journal of Anesthesia. 